\title{
Novel Use of Distal Radius Plates for Wrist Arthrodesis in Upper Limb Spasticity
}

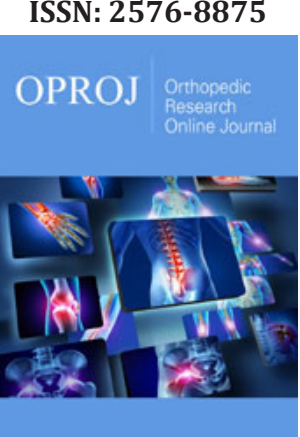

*Corresponding author: Rahul Bamal, Department of Plastic Surgery, Whiston Hospital, Prescot, Merseyside, UK

Submission: 酱 December 01, 2020

Published: 想January 15, 2021

Volume 7 - Issue 5

How to cite this article: Rahul Bamal Paul McArthur. Novel Use of Distal Radius Plates for Wrist Arthrodesis in Upper Limb Spasticity. Ortho Res Online J. 7(5). OPROJ. 000674. 2021. DOI: 10.31031/OPROJ.2021.07.000674

Copyright@: Rahul Bamal, This article is distributed under the terms of the Creative Commons Attribution 4.0 International License, which permits unrestricted use and redistribution provided that the original author and source are credited.

\author{
Rahul Bamal ${ }^{1,2,3 *}$ and Paul McArthur ${ }^{1,2}$ \\ ${ }^{1}$ Department of Plastic Surgery, Whiston Hospital, Prescot, United Kingdom \\ ${ }^{2}$ Department of Plastic Surgery, Alder Hey Children's Hospital, Liverpool, United Kingdom \\ ${ }^{3}$ School of Medicine, Griffith University, Gold Coast, Australia
}

\begin{abstract}
Wrist arthrodesis is commonly performed to improve wrist position, flexion crease hygiene and appearance in patients with spastic upper limbs. These patients often have smaller bones and large commercially available implants lead to complications such as prominence of plate, pain and periprosthetic fractures. Authors hypothesized that Medartis $®$ distal radius plates will lead to lower complication rates on account of lower profile, variable angle locking construct and smaller screw size.
\end{abstract}

A retrospective study included review of upper limb spasticity patients who had undergone wrist arthrodesis under a single lead surgeon with a follow up period of 33 months. Medartis $®$ distal radius volar locking plate was placed dorsally for the wrist fusion surgery along with proximal row carpectomy. 8 wrist arthrodeses were performed in 7 patients using the described technique and implant. The mean age of the patient group was 28 (16-75) years and mean follow up was 15.4 (2-33) months. 91.6 ${ }^{\circ}\left(76^{\circ}-110^{\circ}\right)$ mean improvement in wrist position was achieved after surgery. Fusion was achieved in all cases providing a $100 \%$ fusion rate. Visual Analogue Scale and House scores were used to assess outcomes. Maximum improvement was reported in appearance of the wrist, hygiene and overall satisfaction. There were no periprosthetic fractures and none of the implants had to be removed.

Low profile distal radius volar locking plates can be safely used for wrist fusion in upper limb spasticity with excellent outcomes. For the patient group, the Medartis $®$ distal radius volar locking plate appears to produce superior results with low complication rates when compared to available standard fusion plates used in other series.

Keywords: Wrist arthrodesis; Cerebral palsy; Upper limb spasticity; Distal radius plates

Abbreviations: EPL: Extensor Pollicis Longus; FCR: Flexor Carpi Radialis; FCU: Flexor Carpi Ulnaris;VAS: Visual Analogue Scale

\section{Introduction}

Wrist arthrodesis is commonly performed in patients with upper limb spasticity. The main benefits described in literature are improved wrist position, flexion crease hygiene and appearance [1-4]. Reported disadvantages and associated complications can be prominence of the plate, pain, periprosthetic fractures, malunion and implant loosening $[1,3,5-8]$. Many patients with spasticity have smaller bones for age due to disuse and poor nutrition. The commonly available implants are often designed for arthritic or post-traumatic reconstruction. As such they are larger in size contributing to the associated complications of implant prominence, larger screw sizes leading to periprosthetic fractures and tendon irritation. Some implants are fixed across long metacarpal length leading to metacarpal extension and distal implant prominence associated with pain [1,3,5-8].

The authors hypothesized that lower profile implants with smaller screw size along with avoidance of distal fixation to the middle finger metacarpal will lead to significantly lower complication rates and improved patient compliance. After review of currently available implants we decided on the variable angle locking volar distal radius plates (Medartis $₫$, Basel, Switzerland) for fixation in our spastic wrist arthrodeses surgeries where they are placed dorsally. This paper shares the preliminary results of our experience. The aim of the study was to evaluate any improvement in patient reported outcomes and the incidence of wrist fusion related complications using this device.

\section{Material and Methods}

A retrospective study was conducted that included review of patients operated at Whiston Hospital and Alder Hey Hospital, Liverpool, Merseyside, UK under a single lead surgeon. 
Wrist arthrodesis performed in all cases involved proximal row carpectomy and fixation with the dorsal placement of a distal radius volar locking plate (Medartis ${ }^{\circledR}$, Basel, Switzerland). Follow up was over a time period of 33 months.

\section{Surgical technique}

Patients were positioned supine with the upper limb on an arm table. An upper arm tourniquet was inflated and the operative field was sterilised and draped. Access to the wrist joint was through a dorsal longitudinal incision in line with the middle finger. The third extensor compartment was opened and Extensor Pollicis Longus (EPL) tendon was retracted radially, subperiosteal dissection and supracapsular dissection under the fourth and second compartments was carried out and they were retracted ulnarly and radially respectively using a self retaining retractor. The wrist joint was exposed and a proximal row carpectomy was performed by excision of scaphoid, lunate and triquetrum bones with preservation of bone devoid of cartilage to be used as bone graft. The proximal pole of the capitate was excised, and the hamate and radius were prepared to maximise contact area of raw bone. The wrist was extended and flexor tendon tenotomies or lengthening of Flexor Carpi Radialis (FCR) and Flexor Carpi Ulnaris (FCU) performed depending on individual muscle contractures. A separate volar ulnar longitudinal incision was required to access flexor tendons.

The wrist was brought into a slightly extended position that matched the contour of the plate. Distal screws can be inserted first in order to get at least 3-4 screws into the carpus providing stronger fixation. Once fixed distally then the final proximal fixation was achieved in an acceptable position. A layered, 3-0 monocryl (monofilament absorbable suture) closure with capsule was used to cover implant and extensor tendons. EPL may be left in the subcutaneous plane. Non adherent dressings with a plaster of paris splint for support were applied.

Routinely the wound was reviewed at two weeks postoperative visit and at the same time thermoplastic splint replaced the plaster of paris splint that was provided by the therapist. The wrist was protected in a splint for approximately 6 weeks.

\section{Results and Discussion}

8 wrist arthrodeses were performed using the described surgical technique in 7 patients. 3 patients suffered with cerebral palsy, 1 patient ( 2 wrists) had spasticity following treatment for medulloblastoma, 1 had a stroke and 1 had traumatic brain injury. Indications for surgery were severe fixed wrist flexion deformity interfering with hygiene and daily cares. The mean age of the patient group was 28 (16-75) years and mean follow up was 15.4 (2-33) months. Patients had a pre-operative wrist position of mean flexion $87.7^{\circ}\left(54^{\circ}-10^{\circ}\right)$ (Figure 1). Preoperative positions were reported by patient or parent in most cases while it was missing in one patient. Post-operative wrist position achieved was of a mean $7^{\circ}$ extension $\left(14^{\circ}\right.$ flexion to $50^{\circ}$ extension) as measured on radiographs (Figure 2). $91.6^{\circ}\left(76^{\circ}-110^{\circ}\right)$ mean improvement in wrist position was achieved after surgery. Fusion was achieved in all cases providing a $100 \%$ fusion rate.

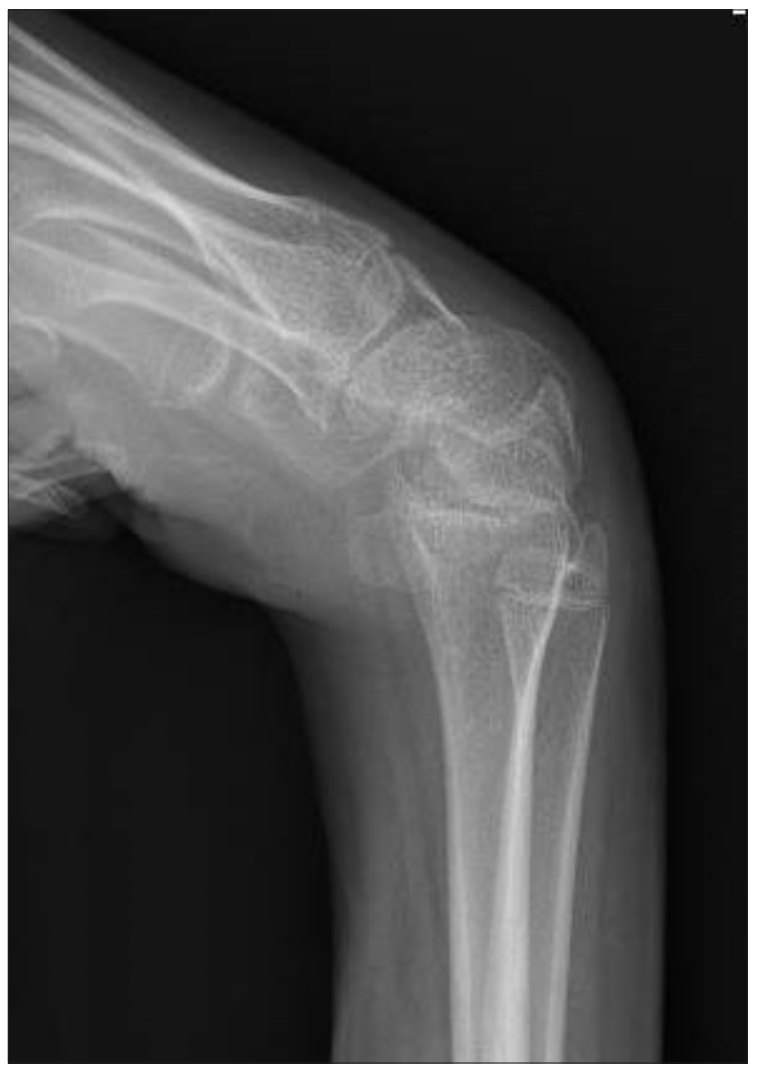

Figure 1: Pre op wrist position showing severe flexion deformity. 


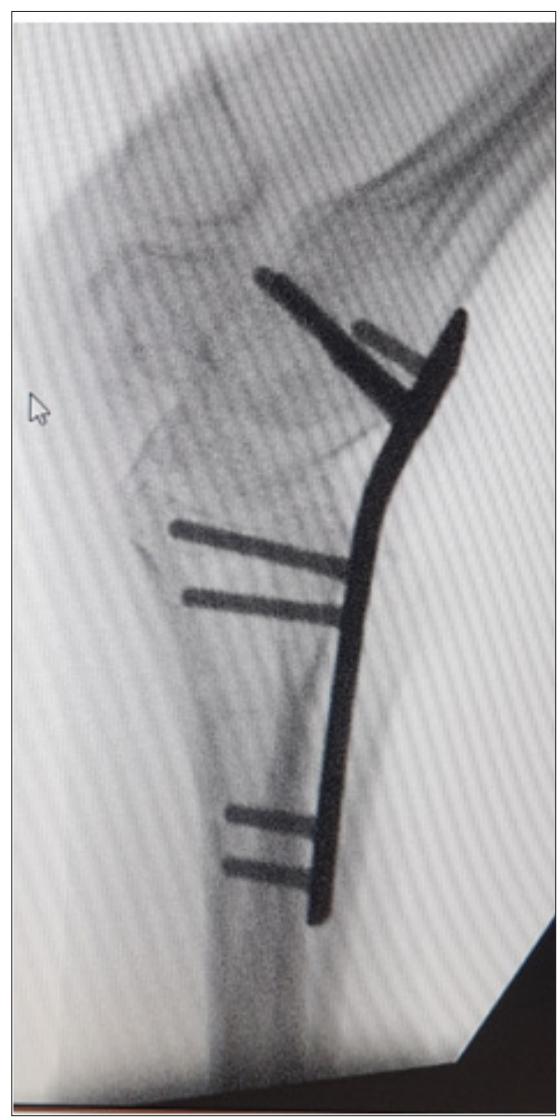

Figure 2: Wrist position post arthrodesis surgery.

All patients had undergone previous spasticity management procedures prior to the arthrodesis. Procedures included intensive therapy (including splints) and botulinum toxin injections, tendon lengthening, tendon releases and tendon transfers. Patient reported outcome scores were used to assess the improvement in a number of parameters after wrist fusion surgery. Visual analogue scale (VAS) and House scores were used as outcome assessment scores $[1,9]$. The scoring was performed by patients themselves or the carer depending on the ability of the patient to communicate. Scores for 7 out of 8 wrists were assessed through a telephone interview in view of coronavirus pandemic restrictions while one assessment was done during a face to face clinic appointment.

Visual Analogue Scale (VAS) involved questions based on improvement in appearance, function, daily cares, hygiene, pain and overall satisfaction. Scores were on a scale of 0-10 with 0 being much worse and 10 being greatly improved. Maximum improvement was reported in appearance of the wrist, hygiene and overall satisfaction while noticeable improvement was reported in the ability to carry out daily cares (Table 1 ). Similar VAS score was used in literature previously [1]. House Score was scored from 0-8 with 0 being no functional use and 8 being complete spontaneous use. Scores did not show improvement in most wrists $(n=6)$ while showed some improvement in only 2 wrists. This implied that wrist fusion does not provide significant improvement in function as also shown by VAS scoring. (Table 1\&2) There were no reports of periprosthetic fractures, non-union, significant implant prominence or pain, tenosynovitis and none of the implants had to be removed. (Table 3) One patient reported worsened thumb in palm deformity due to increased tension in flexor pollicis longus tendon and is being managed by botulinum toxin injections satisfactorily.

Table 1: Visual Analogue scale scores. Pt- patient, Rt- right, Lt- left, X- Not known/scored.

\begin{tabular}{|c|c|c|c|c|c|c|c|c|c|c|}
\hline S.No. & Component & Pt1 & Pt2Rt & Pt2 Lt & Pt3 & Pt4 & Pt5 & Pt6 & Pt7 & Mean \\
\hline 1 & Did surgery improve the appearance of your wrist? & 10 & 10 & 8 & 9 & 4 & 10 & 10 & 8 & 8.63 \\
\hline 2 & Did surgery improve the function of your wrist? & 5 & 0 & 0 & 0 & 0 & 10 & $\mathrm{X}$ & 10 & 3.57 \\
\hline 3 & Has surgery made your daily cares easier? & 7 & 10 & 10 & 0 & 0 & 10 & 5 & 10 & 6.5 \\
\hline 4 & Did the surgery improve your hygiene? & 8 & 10 & 10 & 0 & 8 & 10 & 5 & 10 & 7.63 \\
\hline 5 & $\begin{array}{l}\text { How would you rate your overall satisfaction with sur- } \\
\text { gery? }\end{array}$ & 8 & 5 & 5 & 5 & 7 & 10 & 8 & 10 & 7.25 \\
\hline
\end{tabular}


Table 2: House score for the patients.

\begin{tabular}{|c|c|c|c|}
\hline Patient & Pre-op & Post-op & Change in score \\
\hline 1 & 4 & 7 & 3 \\
\hline 2 Right wrist & 0 & 0 & 0 \\
\hline 2 Left wrist & 0 & 0 & 0 \\
\hline 3 & 7 & 7 & 0 \\
\hline 4 & 0 & 0 & 0 \\
\hline 5 & 1 & 1 & 0 \\
\hline 6 & 0 & 0 & 3 \\
\hline 7 & 0 & 3 & 0 \\
\hline
\end{tabular}

Table 3: Complication rates in comparison with literature.

\begin{tabular}{|c|c|c|}
\hline & This Study & $\begin{array}{c}\text { Van Heest et al. } \\
\text { [1] }\end{array}$ \\
\hline Periprosthetic fracture & 0 & $4(10 \%)$ \\
\hline Implant removal & 0 & $19(46 \%)$ \\
\hline $\begin{array}{c}\text { Prominent/painful } \\
\text { implant }\end{array}$ & 2 (minor prominence) & $11(27 \%)$ \\
\hline Secondary surgery & 0 & $19(46 \%)$ \\
\hline
\end{tabular}

Table 4: Cohort characteristics.

\begin{tabular}{|c|c|c|}
\hline $\mathrm{n}$ & This study & Van Heest et al. [1] \\
\hline $\begin{array}{c}\text { Outcome scores } \\
\text { available (n) }\end{array}$ & 7 prists (7 patients) & 41 wrists (34 patients) \\
\hline Average age & $28(16-75)$ yrs & 23 patients \\
\hline Male: Female & $7: 0$ & $23: 13-50)$ yrs \\
\hline Implant used & $\begin{array}{c}\text { Medartis® volar distal ra- } \\
\text { dius variable angle locking } \\
\text { plate }\end{array}$ & $\begin{array}{c}\text { Synthes } ® ~(A 0) \text { wrist } \\
\text { fusion plate }\end{array}$ \\
\hline Screw size & $2.5 \mathrm{~mm}$ & $\begin{array}{c}2.7 \text { mm distal, 3.5mm } \\
\text { proximal }\end{array}$ \\
\hline Follow up & $15.4(2-33)$ months & $14(2-70)$ months \\
\hline
\end{tabular}

Common challenges in wrist fusion surgery of patients with upper limb spasticity are small bone dimensions and poor bone density due to muscle atrophy and disuse. Current commercially available implants are often too large in size relative to limb size of patients with limb spasticity that are generally young and suffer from cerebral palsy. Larger profile of plates and bigger screw size create problems of implant prominence, periprosthetic fractures and high implant removal rates [1,3,5-8]. Implants used by Van Heest et al had $3.5 \mathrm{~mm}$ proximal screw size and $2.7 \mathrm{~mm}$ distal metacarpal screw size while our implant had much lower profile and screw size of $2.5 \mathrm{~mm}$ placed into the carpus thus avoiding the small metacarpal dimensions (Table 4). The implant used by them was fixed to almost the whole length of middle finger metacarpal distally, thus contributing to complications. Using the variable angle locking screws (and the plating system described in this study) allowed better screw fixation in the carpal region even in osteopenic bone stock (Figure 3-5). Relatively smaller sized $2.5 \mathrm{~mm}$ screws in the radius shaft also appear to reduce complications.

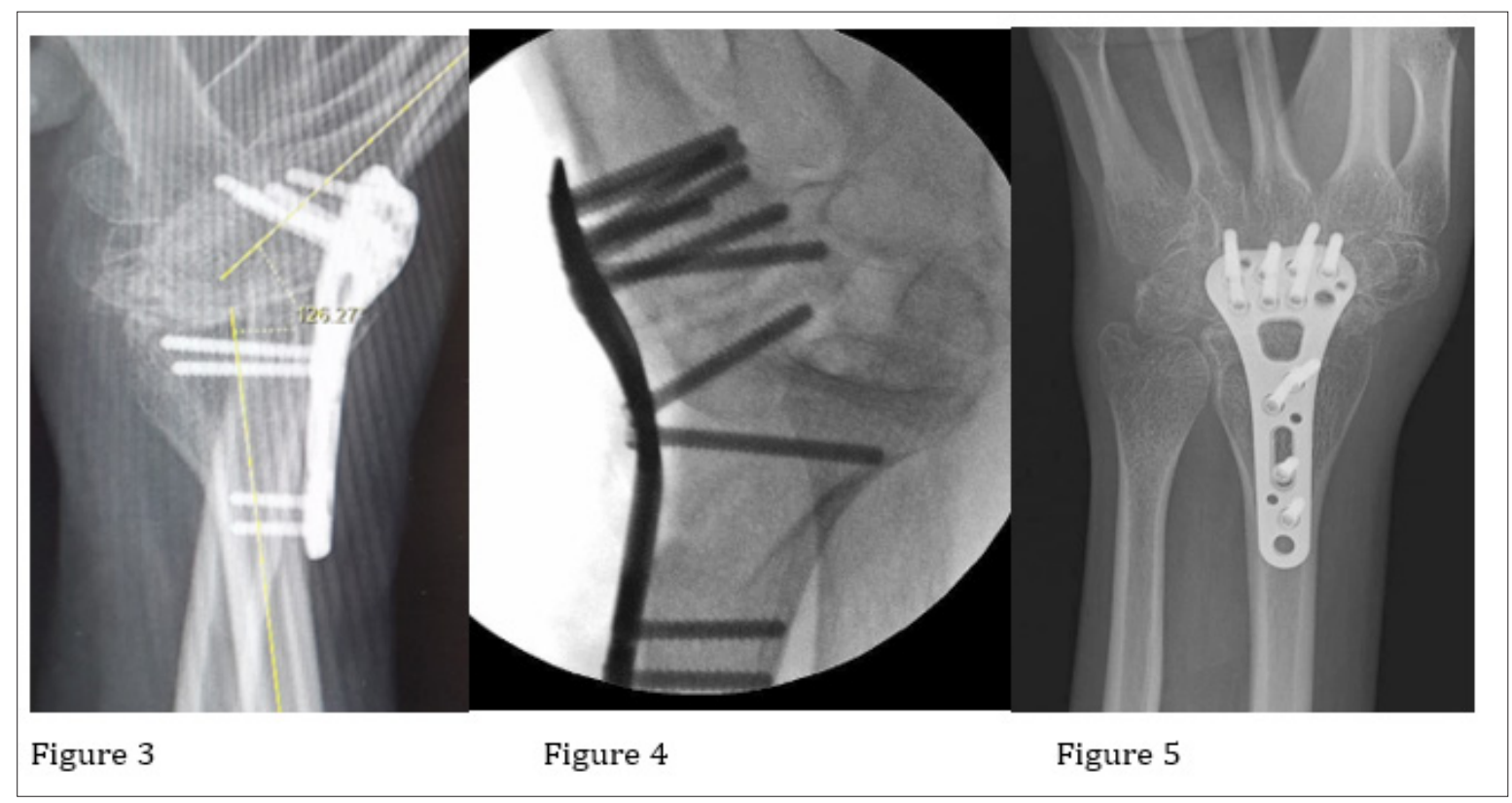

Figures 3-5: Stable construct due to variable angle locking screws in carpal region. 
We used this implant in 8 wrists while a large series had reported their experience in 41 wrists but with similar follow up of 14 months against 15.4 months in our study (Table 4). Outcomes were compared with the findings in the published literature that showed a similar improvement in wrist position, fusion rate and outcome scores (Table 5). Improvement in appearance and hygiene had been consistently noted in various studies on wrist fusion in spastic limbs previously [1-4]. A proximal row carpectomy was performed with wrist arthrodesis in all wrists in this study while it was done in 36 wrists out of 41 by Van Heest et al. [1] A proximal row carpectomy helps to achieve extension by partially overcoming the pull exerted by shortened finger flexors $[2,10]$. It also provides bone graft that can be used for arthrodesis if needed [5,11].

Table 5: Outcome comparison with literature. NA- not available.

\begin{tabular}{|c|c|c|}
\hline $\begin{array}{c}\text { Pre op wrist resting } \\
\text { position }\end{array}$ & This study & Van Heest et al. [1] \\
\hline Post op wrist position & $7^{\circ}$ extension & $86^{\circ}\left(20-135^{\circ}\right)$ \\
\hline $\begin{array}{c}\left(14^{\circ} \text { flexion to } 50^{\circ}\right. \\
\text { extension })\end{array}$ & $\begin{array}{c}5^{\circ} \text { extension } \\
\text { extension })\end{array}$ \\
\hline $\begin{array}{c}\text { Improvement in wrist } \\
\text { position }\end{array}$ & $91.6^{\circ}\left(76^{\circ}-110^{\circ}\right)$ & $89^{\circ}\left(20-148^{\circ}\right)$ \\
\hline Fusion & $100 \%$ & $98 \%$ \\
\hline Outcome Scores: & 8.63 & 7.9 \\
\hline Visual Analogue Scale: & 3.57 & 6 \\
\hline Appearance & 6.5 & 7 \\
\hline Improved function & 7.63 & 6.2 \\
\hline Daily cares & 7.25 & 8.3 \\
\hline Hygiene & & \\
\hline Satisfaction & $1.5(1-7)$ & $0.5(0-2)$ \\
\hline House Score: & $2.25(1-7)$ & $\mathrm{NA}$ \\
\hline pre op & $0.75(0-3)$ & $\mathrm{NA}$ \\
\hline post op & & \\
\hline Change after arthrodesis & & \\
\hline
\end{tabular}

The literature reports a high complication rate with wrist arthrodesis in patients with limb spasticity. [1,5,6,8]. Rates of periprosthetic fractures or implants removed as high as $10 \%$ and $46 \%$ respectively were reported in the literature. [1] The rate of additional surgery due to complications arising from the initial procedure was $39 \%$ and overall rate was $46 \%$. (Table 3 ) Hartigan et al. [5] and Zachary et al. [6] reported $8-12 \%$ periprosthetic fracture rates. Approximately 25\% implant removal rate was reported by Zachary et al. [6] and 0'Bierne et al. [8] In this series we report low complication rate and there have been no implant related complications. We consider that the implant achieved sufficient anchorage in the carpals and radius despite often poor bone quality in this patient group.

\section{Limitations}

Limitations of this study are retrospective nature, a small sample size, possibility of recall bias in patient reported outcomes and no control group or randomisation of patients. The limitations imposed by the covid pandemic did not allow face to face review of the patients in most cases.

\section{Conclusion}

Low profile distal radius volar locking plates can be safely used for wrist fusion in upper limb spasticity with excellent outcomes. The plates are used on the dorsal aspect of the radius and carpus and, to date have shown positive results. Variable angle locking screws help to achieve a stable construct in spite of poor bone stock that is often found in patients with spasticity due to osteopenia and disuse atrophy. The complication rate in this study is low as compared to other reported series using different plating systems for this condition. For the patient group the Medartis ${ }^{\circledR}$ distal radius volar locking plate appears to produce superior results when compared to available standard fusion plates used in other series. We consider that this is due to the implant characteristics and surgical technique. None were required to be removed in the patient group when used for wrist fusion.

\section{Acknowledgement}

We acknowledge the Department of Plastic Surgery, Whiston Hospital, UK for covering the publication fee.

\section{Conflict of Interest}

No financial interest or any conflict of interest to be declared.

\section{References}

1. Van Heest, Ann E (2009) Wrist arthrodesis in cerebral palsy. Journal of Hand Surgery 34(7): 1216-1224.

2. Hoffer MM, Zeitew S (1988) Wrist fusion in cerebral palsy. J Hand Surg 13(5): 667-670.

3. Pomerance JF, Keenan MA (1996) Correction of severe spastic flexion contractures in the nonfunctional hand. J Hand Surg 21(5): 828-833.

4. Rayan GM, Young BT (1999) Arthrodesis of the spastic wrist. J Hand Surg 24(5): 944-952.

5. Hartigan BJ, Nagle DJ, Foley MJ (2001) Wrist arthrodesis with excision of the proximal bones using the AO/ASIF wrist fusion plate and local bone graft. J Hand Surg 26(3): 247-251.

6. Zachary SV, Stern PJ (1995) Complications following AO/ASIF wrist arthrodesis. J Hand Surg 20(2): 339-344.

7. Alexander, Randall D, Davids, Jon R (2000) Wrist arthrodesis in children with cerebral palsy. Journal of Pediatric Orthopaedics 20(4): 490-495.

8. O'Bierne J, Boyer MI, Axelrod TS (1995) Wrist arthrodesis using a dynamic compression plate. J Bone Joint Surg 77(5): 700-704.

9. Van Heest AE, House J, Cariello C (1999) Upper extremity surgical treatment of cerebral palsy. J Hand Surg Am 24: 323-330.

10. Hargreaves DJ, Warwick DJ, Tonkin MA (2000) Function following wrist arthrodesis in cerebral palsy. The Journal of Hand Surgery: British \& European 25(2): 193-194.

11. Richards RS, Roth JH (1994) Simultaneous proximal row carpectomy and radius to distal carpal row arthrodesis. J Hand Surg 19(5): 728-732.

For possible submissions Click below: 